\title{
Aplicação da lâmpada de descarga de mercúrio sem eletrodo para degradação do paracetamol
}

\author{
Ailton Jose Moreira ${ }^{*}$; Lilian Oliveira Campos ${ }^{\mathrm{b}}$, Gian Paulo Giovanni Freschic \\ a Programa de Pós-graduação em Química, Universidade Federal de Alfenas, Poços de Caldas, 37715-400, Minas Gerais, Brasil. \\ ${ }^{b}$ Laboratório de Fotólise, Fotocatálise e Especiação Química, Universidade Federal de Alfenas, Poços de Caldas, 37715-400, Minas \\ Gerais, Brasil. \\ c Programa de pós-graduação em Química, Universidade Federal de Alfenas, Poços de Caldas, 37715-400, Minas Gerais, Brasil. \\ *aijomoquim@gmail.com
}

Recebido: 16 julho 2018 / Aceito: 9 setembro 2018 / Publicado online: 12 setembro 2018

\begin{abstract}
Resumo
Lâmpada de descarga de mercúrio sem eletrodo (Hg-EDL) foi aplicada em estudos de degradação fotolítica e fotocatalítica para avaliar o potencial de degradação de uma solução de Paracetamol $10 \mathrm{mg} \mathrm{L}^{-1}$. A fotodegradação (fotólise e fotocatálise) foi conduzida em reator UV/MW, com potência microondas fixa $(200 \mathrm{~W}, \mathrm{pH} 7)$ e diferentes tempos de irradiação $(0,083$ a 2,0 min.). Após irradiadas, as amostras foram analisadas através de espectrofotometria UV/Vis para quantificação do fármaco. Houve a remoção de até $70,1 \%$ no ensaio fotolítico com o tempo de 2 min., enquanto para o processo fotocatalítico (aplicando suspensão de $1 \mathrm{~g} \mathrm{~L}^{-1}$ dos semicondutores) os resultados de remoção foram da ordem de $60 \%$. A cinética de primeira ordem foi aplicada, sendo determinada uma constante $\mathrm{k}=0,602 \mathrm{~min}^{-1} \mathrm{e}^{2}=0,993$, evidenciando um ajuste adequado a cinética de ordem 1. Assim, o reator UV/MW demonstra elevada eficiência no processo de degradação do paracetamol, uma vez que elevada taxa de remoção e valor elevado de constante cinética de degradação foram obtidas para o respectivo sistema. $\mathrm{O}$ foto reator avaliado pode ser explorado em ensaios de degradação com outros compostos, possibilitando a avaliação de sua eficiência frente a degradação de uma maior variedade de contaminantes emergentes.
\end{abstract}

Palavras-chave: Processos Oxidativos Avançados, fotoquímica, poluentes emergentes, fármacos.

\section{Application of mercury discharge lamp without electrode for degradation of paracetamol}

\begin{abstract}
Electroless mercury discharge lamp (Hg-EDL) was applied in photolytic and photocatalytic degradation studies, to evaluate the degradation potential of a solution of Paracetamol $10 \mathrm{mg} \mathrm{L}^{-1}$. The photodegradation (photolysis and photocatalysis) was conducted in a UV / MW reactor, with fixed microwave power (200W, $\mathrm{pH} \sim 7)$ and different irradiation time $(0.083$ and 2.0 min.). After irradiation, the samples were analyzed by UV / Vis spectrophotometry for drug quantification. Removal of up to $70 \%$ in the photolytic assay with time of up to $2 \mathrm{~min}$., while for the photocatalytic process (applying suspension of $1 \mathrm{~g} \mathrm{~L}^{-1}$ of the semiconductors) the results removal was of the order of $60 \%$. First-order kinetics were applied, with a constant $\mathrm{k}=0.602$ $\min ^{-1}$ and $\mathrm{r}^{2}=0.993$, demonstrating an adequate adjustment to the kinetics of order 1 . Thus, the UV / MW reactor demonstrates high efficiency in the paracetamol degradation process, since high removal rate and high kinetic degradation value were obtained for the respective system. This photoreactor can be explored in degradation tests of other compounds, allowing the evaluation of its efficiency against the degradation of a greater variety of emerging contaminants.
\end{abstract}

Keywords: Advanced Oxidative Processes, photochemistry, emerging pollutants, drugs.

\section{Introdução}

O crescimento econômico das indústrias farmacêuticas, trouxeram como consequência, quadros de contaminação atmosférica, do solo e dos recursos hídricos em todo o mundo através de moléculas orgânicas. A qualidade da água tem sido extensivamente discutida, tendo em vista que se trata de um recurso natural imprescindível a um largo espectro de atividades humanas, onde se destacam, entre outros, o abastecimento público e industrial. Diante dessa conjuntura, temas como reuso, minimização e tratamento de resíduos vêm ganhando cada vez mais importância (Melo et al., 2009).

Geralmente, os fármacos são absorvidos pelo organismo e estão sujeitos a reações metabólicas. Entretanto, uma quantidade significativa dessas substâncias e seus metabólitos são excretados na urina, fezes ou esterco animal, sendo frequentemente encontrados no esgoto doméstico (Bila e 
Dezotti, 2003).

Visto que o tratamento de efluente convencional não é $100 \%$ eficaz para degradação de contaminantes emergentes, e levando em consideração que, apenas 50\% do esgoto gerado no Brasil, possui sistema de coleta e, que destes, apenas $40,8 \%$ passa por algum processo de tratamento de efluentes (Brasil, 2016), a situação se agrava, trazendo consequências para o meio ambiente. Devido ao tempo de persistência dos compostos e, seus riscos à saúde e ao meio ambiente, alguns estudos tem buscado processos mais rápidos e eficientes para degradação desses contaminantes e, dentre os métodos estudados, os processos oxidativos avançados (POAs) se destacam (Tong et al., 2012; Barros, 2014). Os Processos Oxidativos Avançados, são processos que se baseiam na formação de radicais livres, principalmente o radical hidroxila $\left({ }^{\circ} \mathrm{OH}\right)$, altamente reativos e pouco seletivos (Fioreze, 2014; Ta et al., 2016).

Em 1953, a Companhia Sterling-Winthrop lançou o paracetamol no mercado como um fármaco analgésico e antitérmico, e desde então, passou a ser um dos medicamentos mais consumidos no mundo (Domingos, 2010).

Segundo (Pereira, 2018), foi constatado a presença de paracetamol em rios e lagos, tanto no Brasil, quanto em outros países, com concentrações na ordem de $\mu \mathrm{g} \mathrm{L}^{-1}$. Algumas das consequências que o paracetamol resulta aos seres vivos, é que quando ingerido em altas doses, pode ser nocivo ao fígado humano e dos animais, e em doses pequenas, por exemplo, quando ingerida por grávidas, pode causar aos seus filhos, contribuições para surgimento de alergias. Deste modo, observa-se que, mesmo em concentrações baixas, o fármaco pode trazer danos para os seres humanos e, outros estudos mostram que este, também afeta a saúde dos animais (Napoleão, 2015).

A importância do presente estudo, esta atribuída a necessidade que a sociedade moderna possui em possibilitar o desenvolvimento de tecnologias mais eficientes para tratamento de poluentes emergentes, uma vez que, os métodos atualmente aplicados, não se apresentam efetivos para remoção destes compostos em águas e efluentes.

Essa pesquisa teve como objetivo, avaliar o potencial de degradação fotolítico e fotocatalítico do paracetamol, sob exposição as energias microondas e ultravioleta (UV/MW) aplicando-se variação de alguns parâmetros físico-químicos para otimização do sistema de degradação.

\section{Metodologia}

Os estudos de degradação do paracetamol foram conduzidos no reator UV/MW (MARS $6,220 \mathrm{~V}, 60 \mathrm{~Hz}, \mathrm{CEM}$, Matthews, NC,EUA), sendo utilizados $10 \mathrm{~mL}$ de uma solução aquosa de paracetamol na concentração de $10 \mathrm{mg} \mathrm{L}^{-1}(\mathrm{pH} \sim 7)$, sendo este volume transferido para a lâmpada $\mathrm{Hg}$-EDL (UMEX GmbH, Dresden, Alemanha) e submetido a irradiação no intervalo de tempo de até 2 min., sendo a lâmpada acionada sob potência microondas fixa de $200 \mathrm{~W}$. Reagente sequestrante de radical hidroxila (Dimetilsulfóxido (DMSO)) foi aplicado junto a solução de paracetamol $10 \mathrm{mg}$ $\mathrm{L}^{-1}$, na proporção de $1 \%\left(\mathrm{v} \mathrm{v}^{-1}\right)$, permitindo avaliar sua influência no processo de degradação.
Para o estudo de influência do $\mathrm{pH}$, soluções aquosas de paracetamol $10 \mathrm{mg} \mathrm{L}^{-1}$ foram preparadas com ajuste de $\mathrm{pH}$ equivalente a 2 - 5- 7 -9 - 11. Para o ajuste, foi utilizada solução de $\mathrm{HCl}$ e/ou $\mathrm{NaOH} 0,1 \mathrm{~mol} \mathrm{~L}^{-1}$.

Uma alíquota da solução foi submetida a análise espectrofotômetrica UV-Vis Cary 60 após as irradiações, permitindo a quantificação do fármaco, sendo estas determinações, executadas em triplicata. As mesmas alíquotas foram submetidas a leitura de $\mathrm{pH}$, a fim de obter uma melhor compreensão dos mecanismos de degradação do paracetamol. $\mathrm{O} \mathrm{pH}$ foi medido por meio de análise potenciométrica, com eletrodo de membrana de vidro e potenciômetro Metrohm 827 pH Lab (Metrohm, São Paulo, SP, Brasil).

As soluções foram submetidas aos ensaios fotocatalíticos (na presença do semicondutor $\mathrm{TiO}_{2}$ e $\mathrm{TiO}_{2}$ dopado com Boro $\left(\mathrm{TiO}_{2}-\mathrm{B}\right)$, obtidos através de síntese sonoquímica e caracterizados com maior percentual da sua fase fotoativa, anatase). Anteriormente a etapa de irradiação, as amostras foram agitadas por período de $2 \mathrm{~min}$. (etapa preliminar de adsorção) e posteriormente irradiadas. Após o período de irradiação, as amostras foram conduzidas a analise espectrofotométrica. Membrana filtrante de $0,22 \mu \mathrm{m}$ foram aplicadas para remoção do semicondutor. A agitação não foi possível durante a degradação, porém, as amostras eram devidamente agitadas por período de 2 min., antes de transferidas a lâmpada $\mathrm{Hg}$-EDL, permitindo melhor dispersão do semicondutor na extensão da solução aquosa de paracetamol. Salienta-se que, os períodos de exposição foram de no máximo 2 min., e considerando que a radiação se propaga em todas as direções do reator (incide em todas as posições da amostra), mesmo sem a agitação durante o tempo de irradiação (0,083 a 2,0 min.), o aproveitamento energético é considerado adequado.

Para quantificação do paracetamol (antes e após os ensaios de fotodegradação), uma curva de calibração através de ensaios de absorção molecular foi processada. As soluções com concentração conhecida de paracetamol $\left(0,3\right.$ a $\left.20,0 \mathrm{mg} \mathrm{L}^{-1}\right)$ tiveram suas absorbâncias monitoradas na região de $243 \mathrm{~nm}$, possibilitando a obtenção da equação linear da curva de calibração. Assim, a quantificação de paracetamol foi conduzida através da equação [paracetamol] $\left(\mathrm{mg} \mathrm{L}^{-1}\right)=$ (Absorbância - 0,00756)/0,0674, obtida após ensaios com soluções padrões de concentração conhecida, apresentando $\mathrm{r}^{2}$ $=0,996$.

Para caracterização espectral e energética do fotoreator, foi utilizado um espectrorradiômetro SPR-4002 (Luzchem, Ottawa, Ontario, Canada), com faixa de leitura espectral de 230 a $900 \mathrm{~nm}$, cobrindo as regiões UVA, UVB, UVC e visível. Os espectros de radiação foram quantificados através da intensidade em $\mathrm{mW} \mathrm{m}^{-2}$. O detector do espectrorradiômetro foi posicionado na parte superior do microondas, sendo as emissões eletromagnéticas provindas da lâmpada Hg-EDL, coletadas por período de tempo de $1 \mathrm{~min}$., em triplicata.

\section{Resultados e Discussão}

Caracterização espectral e energética do fotoreator UV/MW

As medidas espectroradiométrica evidenciam que o fotoreator UV/MW, emite radiação nos comprimentos de onda 
254 - 314 - 366 - 407 - 437 - 547 e 579 nm, sendo estes, atribuídos as regiões ultravioleta e visível. De acordo com (Církva e Relich, 2011), estas regiões de emissão espectral são verificadas para as lâmpadas $\mathrm{Hg}-\mathrm{EDL}$, sendo caracterizadas pelo autor, como espectros de emissão mais limpos, contribuindo de modo positivo para as reações fotoquímicas que são conduzidas no respectivo sistema.

Considerando que compostos orgânicos são absorvedores de radiação UV/Vis (Kemary et al., 2011; Martignac et al., 2013), e que no caso do paracetamol, este possui capacidade de absorção mais elevada nos comprimentos de onda próximos a região UV $(243 \mathrm{~nm})$, como constatado através de medidas de absorção molecular, podemos afirmar que, as emissões espectrais associadas a região visível do reator UV/MW não exercem efeitos significativos no processo de degradação fotolítica e fotocatalítica do respectivo fármaco, e deste modo, a maior contribuição está associada as emissões na região UV, como também é verificado na literatura (Yavas e Ince, 2016; Xiong e Hu, 2012).

Importante destacar que, as intensidades de emissão verificadas correspondem a $4 \mathrm{~W} \mathrm{~m}^{2}(254 \mathrm{~nm}), 2,8 \mathrm{~W} \mathrm{~m}^{2}(314$ $\mathrm{nm}), 6,3 \mathrm{~W} \mathrm{~m}^{2}(367 \mathrm{~nm})$ e até $68,1 \mathrm{~W} \mathrm{~m} \mathrm{~m}^{2}(547 \mathrm{~nm})$ para os principais espectros verificados na região UV e Visível, sendo esta tendência verificada para sistemas parecidos, que são relatados em estudos (Wang et al., 2018; Hong et al., 2015). Mesmo a intensidade de emissão para a região UV $(254 \mathrm{~nm})$, sendo apenas $4 \%$ da intensidade observada para a região visível $(547 \mathrm{~nm})$, é necessário considerar que os espectros UV são mais energéticos, e de maior potencial de absorção pelo fármaco, como comprovado através de seu espectro de absorção molecular.

Ainda sobre as características do reator aplicado no presente estudo, é de grande relevância destacar que, as paredes externas da lâmpada Hg-EDL são constituídas de quartzo de baixa permeabilidade ao UV, enquanto que as suas paredes internas (onde se encontra o compartimento de amostra) são permeáveis ao UV. Estas características permitem que, a radiação UV emitida pelo sistema, se concentre no centro do respectivo reator, onde se localiza a amostra, e deste modo, o aproveitamento energético é máximo, permitindo a incidência quase que total da radiação sobre a solução contendo o respectivo fármaco.

\section{Degradação fotolítica do paracetamol}

Os estudos de influência do $\mathrm{pH}$ inicial $\left(\mathrm{pH}_{\mathrm{O}}\right)$ foram conduzidos para constatação das melhores condições de degradação do paracetamol. Assim, os resultados apresentados na Figura 1, evidenciam que a influência do pHo frente a degradação do paracetamol não é significativa, e deste modo, processos de controle de pHo para os ensaios de degradação não se fazem necessários.

Também é verificado através da Figura 1B que, para os diferentes valores de $\mathrm{pHo}$, constata-se que o $\mathrm{pH}$ (final) da solução apresenta decaimento com o decorrer do tempo de degradação, ou seja, a degradação do paracetamol promove o aumento da concentração de íons $\mathrm{H}^{+}$no sistema, e deste modo, a desprotonação é um dos mecanismos de degradação do paracetamol.

Estudos conduzidos por (Trovó et al., 2012), durante a degradação do paracetamol, quantificou a evolução de ácidos carboxílicos, tais como, ácido fórmico, acético, propriônico e oxálico.
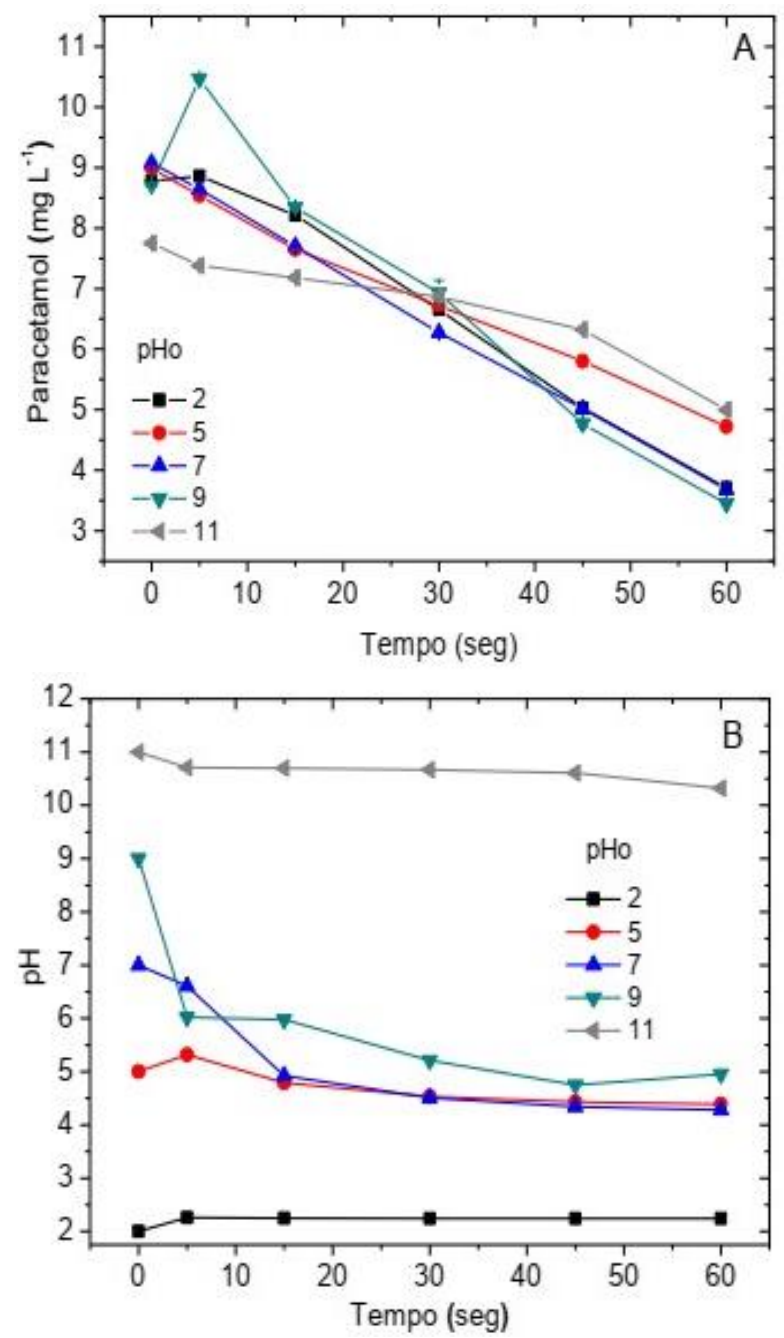

Figura 1. Variação da concentração de paracetamol em diferentes condições de pH inicial - pHo (A) e pH após processo degradação fotolítica (B).

Estudos de degradação do paracetamol por processos oxidativos avançados tem sido abordado em pesquisas científicas, tais como, fotodegradação com lâmpada xênon à $300 \mathrm{~nm}$ (Bai, 2018), sonólise e sonofotocatálise (Yavas, 2015) e fotocatálise (Xiong e Hu, 2012), sendo que variações de $\mathrm{pH}$ após o processo de degradação, como apresentados no presente estudo, tem sido confirmadas. Os sistemas de fotodegradação aplicados nos estudos citados, permitiram degradação de até $85 \%$ do paracetamol em período de $24 \mathrm{~h}$, tempo este, que representa um valor 720 vezes superior ao maior tempo de irradiação $(2 \mathrm{~min})$ aplicado para o reator utilizado nesta pesquisa.

A aplicação do sistema fotolítico $\mathrm{Hg}$-EDL foi conduzido para a degradação de outro contaminante emergente (atrazina) (Moreira et al., 2017), e os resultados de elevada eficiência nas taxas de remoção reforçam que, sua atuação em processos avançados de oxidação é interessantes não apenas para uma classe de contaminantes emergentes, mas também para classes distintas. 
Para as amostras expostas a tempos de irradiação de até 2 minutos, observou-se 70,1\% de degradação do paracetamol em 2 minutos de exposição e, a amostra contendo DMSO, a remoção não foi evidente, como ilustra a Figura $2 \mathrm{~A}$.
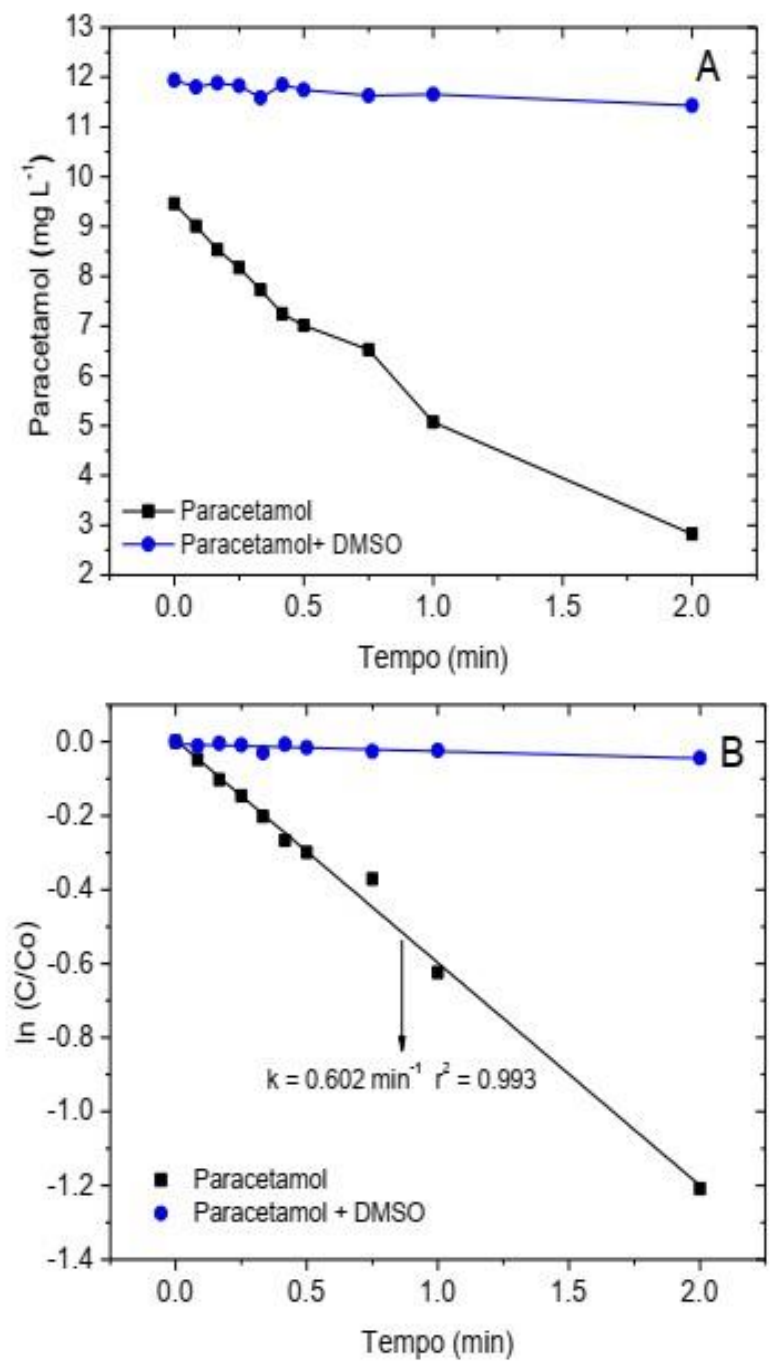

Figura 2. Degradação do Paracetamol em função do tempo (A), e cinética de degradação (B), com e sem Dimetilsulfóxido (DMSO). In (C/Co): índice da relação entre a concentração final (C) e inicial (Co) de paracetamol.

A constante cinética de degradação (ordem 1) obtida no presente estudo $\left(\mathrm{k}=0,602 \mathrm{~min}^{-1}\right)$ apresenta valor $85 \%$, superior ao obtido por (Valdez et. al; 2012), que ao estudar a fotodegradação do Paracetamol através da eletrocatálise, obteve uma constante cinética de ordem 1 , equivalente a $\mathrm{k}=$ $0,09 \mathrm{~min}^{-1}$. Quando adicionado DMSO na condição de agente sequestrante de radical hidroxila (Treml e Smejkal, 2016), a degradação do paracetamol é inibida, possivelmente devido ao sequestro da radical hidroxila pelo DMSO (Figura 2B).

Após serem degradadas os resultados de medida de $\mathrm{pH}$ para as amostras, evidenciam que a desprotonação é um processo ativo no mecanismo de degradação do paracetamol, sendo os dados apresentados através da Figura 3.

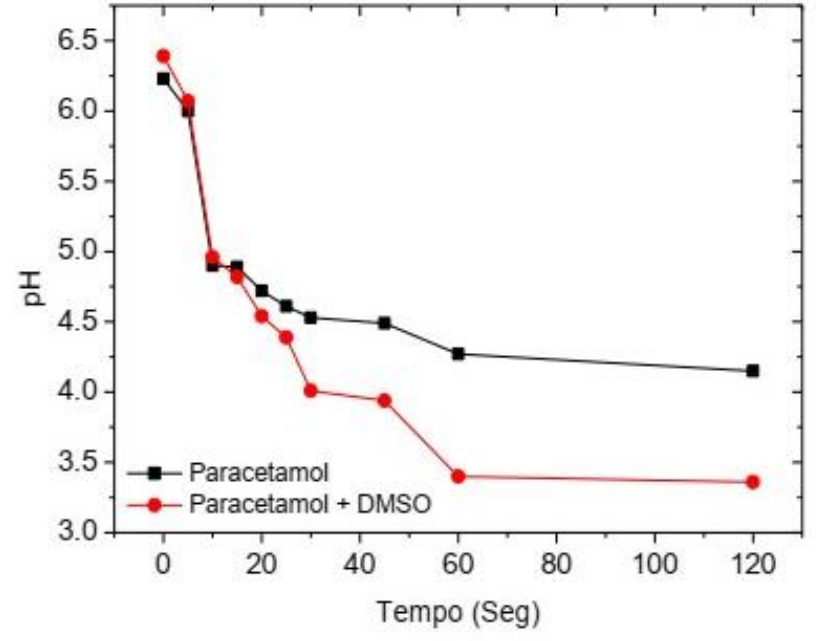

Figura 3. Variação do $\mathrm{pH}$ das soluções irradiadas de paracetamol em função do tempo de exposição, com e sem Dimetilsulfóxido (DMSO).

Avaliando os dados apresentados é possível perceber que ambas as curvas apresentaram um decréscimo do $\mathrm{pH}$ no começo da degradação, evidenciando a desprotonação ativa (Figura 3).

Após 25 segundos de irradiação, o processo de desprotonação apresenta valores que correspondem a $81 \%$ de equivalência, quando o processo com DMSO é comparado ao processo sem DMSO, e este resultado, pode ser atribuído a formação do ácido metano sulfínico (Steiner et al. 1990), que é resultado da reação entre radicais hidroxila e DMSO.

Após o período de 60 segundos de irradiação, a variação de pH apresenta valores de 0,12 (Sem DMSO) e 0,03 (com DMSO) representando $3 \%$ e $1 \%$ de variação de $\mathrm{pH}$ para um período de 60 segundos, respectivamente, e deste modo, a inibição da desprotonação pode ser afirmada.

De modo geral, na etapa de fotólise conduzida junto a reator UV/MW, confirma-se que o sistema apresenta elevada efetividade na taxa de remoção do paracetamol, onde valores acima de $70 \%$ é alcançado para curtos períodos de irradiação (2 min). Os processos avançados de oxidação podem ainda ser otimizados quando um semicondutor é adicionado ao sistema, e deste modo, a avaliação da inserção de $\mathrm{TiO}_{2}$, puro e dopado com Boro, foi executada no presente projeto, avaliando a atuação da fotocatalise frente a degradação do paracetamol.

Para efeito de comparação, a curva de degradação do paracetamol através da fotólise e fotocatalise estão apresentadas na Figura 4.

O estudo de degradação fotocatalítica utilizando os semicondutores de $\mathrm{TiO}_{2}$ não apresentaram melhora no processo de degradação, sendo que, a diferença entre o processo fotolítico e fotocatalítico não é significativo. Vale destacar que, para o processo fotocatalítica, a remoção apresenta valor $10 \%$ inferior ao verificado para o processo fotolítico. Deste modo, para o presente estudo a inserção dos semicondutores pode ser dispensada, uma vez, que as condições otimizadas da fotocatálise não foram exploradas na etapa de aplicação. 


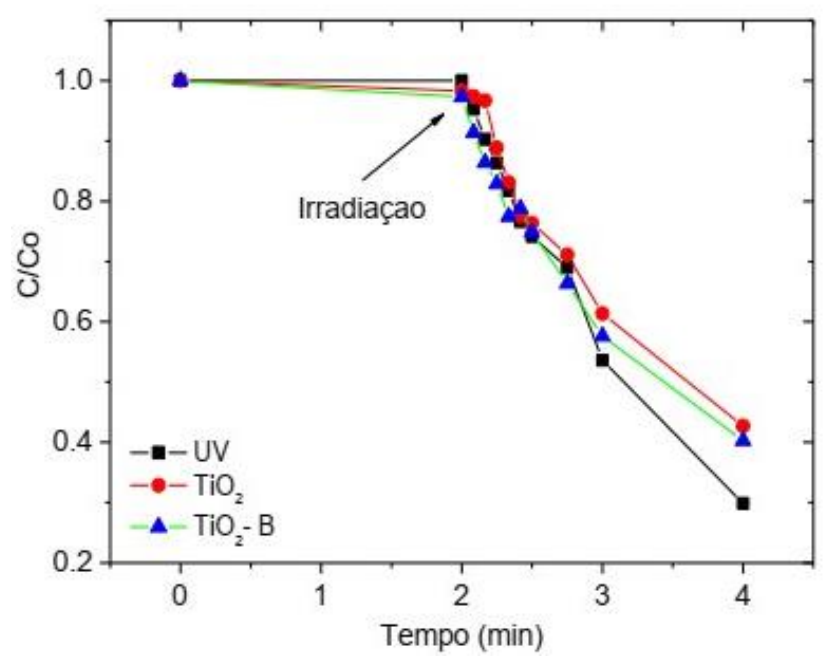

Figura 4. Curva de degradação do paracetamol através do processo fotolítico (UV), fotocatalítico $\mathrm{TiO}_{2}$ e $\mathrm{TiO}_{2}-\mathrm{B}$. $\mathrm{C} / \mathrm{Co}$ : índice da relação entre a concentração final (C) e inicial (Co) de paracetamol.

Importante destacar que, os processos de degradação fotocatalítica podem ser otimizados através de etapas de ajuste de pHo da solução, massa de semicondutor a ser utilizada, tipo de radiação aplicada e controle do tamanho de partícula obtida. Deste modo, para o presente estudo, estas condições não foram otimizadas, uma vez que elevadas taxas de remoção foram alcançadas para o processo fotolítico, porém, para estudos futuros, estes parâmetros deverão ser avaliados.

\section{Conclusões}

A elevada taxa de degradação do paracetamol (acima de $85 \%$ ) em até 2,0 min, sugere que o mecanismo de fotodegradação ocorre por meio de oxidação radicalar (Radicais Hidroxila), visto que, após a adição de DMSO (agente sequestrante de radical hidroxila), a taxa de degradação foi diminuída. A elevada eficiência da fotólise pode ser comprovada em função da constante cinética de degradação do paracetamol $\left(\mathrm{k}=0,602 \mathrm{~min}^{-1}\right)$, que é considerada elevada quando se compara com valores da literature.

A aplicação dos semicondutores não possibilitou a melhora no processo de degradação, porém, esta eficiência pode ser otimizada quando parâmetros físico-químicos controlados forem aplicados na etapa de degradação, e deste modo, estas etapas podem ser aplicadas em estudos futuros. Esse sistema de fotodegradação pode ser otimizado para processos de degradação do paracetamol, onde alguns parâmetros físico-químicos podem ser avaliados, tais como, a influência da concentração inicial, influência da variação da potência microondas e inserção de agentes oxidantes (peróxidos, persulfato).

\section{Agradecimentos}

À Fundação de Amparo à Pesquisa do Estado de Minas Gerais (FAPEMIG) e à Coordenação de Aperfeiçoamento de Pessoal de Nível Superior (CAPES) pelo auxílio financeiro.

\section{Referências}

Barros, A.L. 2014. Estudos de Degradação de fármacos em meio aquoso por processos oxidativos avançados. $100 \mathrm{f}$. Tese (Doutorado). Universidade Federal do Ceara, Fortaleza.

Bai, Y.; Cui, Z.; Su, R.; Qu, K. 2018. Influence of DOM components, salinity, $\mathrm{pH}$, nitrate, and bicarbonate on the indirect photodegradation of acetaminophen in simulated coastal Waters. Chemosphere, 205(Ago.):108-117.

Bila, D.M.; Dezotti, M. 2003. Fármacos no Meio Ambiente. Química Nova, 26(4): 523-530.

Brasil. 2016. Diagnóstico dos Serviços de Água e Esgotos - 2014. Ministério das Cidades. Secretaria Nacional de Saneamento Ambiental - SNSA. Sistema Nacional de Informações sobre Saneamento e Ministério das Cidades - SNSA/MCIDADES), Brasília:, 212p. Disponível: http://www.epsjv.fiocruz.br/upload/Diagnostico_AE2014.pdf. Acesso: 20 jul. 2018.

Církva, V.; Relich, S. 2011. Microwave Photochemistry and Photocatalysis. Part 1: Principles and Overview. Current Organic Chemistry, 15(2): 248264

Domingos, H. 2010. Paracetamol, $\mathrm{C}_{8} \mathrm{H}_{9} \mathrm{NO}_{2}$. Química Nova Interativa. 2010. Disponível

<http://qnint.sbq.org.br/qni/popup_visualizarMolecula.php?id=s8vaLIMvEcKje92Uy3Cfc8eNyEiSRdkk4cMCgowU3EzY8naVq8xJfzI9iazVJSF2Bvg7he8U2DEISQ_hG5UQ>. Acesso em: 19 jul. 2018.

Fioreze, M.; Santos, E.P. Schmachtenberg, N. 2014. Processos oxidativos avançados: fundamentos e aplicação ambiental. Revista Eletronica em Gestão, Educação e Tecnologia Ambiental, 18(1): 79-91.

Hong, J.; Han, B.; Yuan, N.; Gu, J. 2015 The roles of active species in photodecomposition of organic compounds by microwave powered electrodeless discharge lamps. Journal of Envronmental Science, 33(Jul.): 60-68.

Kemarya, M.E.; Sobhya, S.; Daly, S.E.; Shafic, A.A. 2011. Inclusion of Paracetamol into - cyclodextrin nanocavities in solution and in the solid state. Spectrochimica Acta Part A, 79 (5): 1904-1908.

Martignac, M.; Oliveros, E.; Maurette, M.T.; Claparols, C.; Marquié, F.B. 2013. Mechanistic pathways of the photolysis of paracetamol in aqueous solution: an example of photo-Fries rearrangement. Photochem. Photobiol. Sci., 12(3): 527-535.

Melo, S.A.S.; Trovó, A.G.; Bautitz, I.R.; Nogueira, R.F.P. 2009. Degradação de fármacos residuais por processos oxidativos avançados. Química Nova, São Paulo, 32(1): 188-197.

Moreira, A.J.; Borges, A.C.; Gouveia, L.F.C.; Macleod, T.C.O., Freschi, G.P.G. 2017. The process of atrazine degradation, its mechanism, and the formation of metabolites using UV and UV/MW photolysis. Journal of Photochemistry and Photobiology A: Chemistry 347(1): 160-167.

Napoleão, D.C.; Zaidan, L.E.M.C.; Salgado, J.B.A.; Sales, R.V.L.; Silva, V.L. 2015. Degradação do Contaminante Emergente Paracetamol Empregando Processos Oxidativos Avançados. Revista Eletrônica em Gestão, Educação e Tecnologia Ambiental Santa Maria, 19(3): 725-734.

Pereira, B.V.R. 2018. Efeito agudo e crônico dos fármacos Paracetamol e Propranolol em diferentes biomarcadores de uma espécie de peixe neotropical. 100 f. Dissertação de Mestrado. Universidade Federal de São Carlos, Sorocaba.

Steiner, M.G.; Babbs, C.F. 1990. Quantitation of the Hydroxyl Radical by Reaction with Dimethyl Sulfoxide. Archives of biochemistry and biophysics, 278(2), $478-481$.

Ta, N.; Hong, J.; Liu, T.; Sun, C. 2016. Degradation of atrazine by microwaveassisted electrodeless discharge mercury lamp in aqueous solution. Journal of Hazardous Materials, 138(1): 187-194.

Tong, A.Y.C.; Braund, R.; Warren, D.S.; Peake, B.M. 2012. $\mathrm{TiO}_{2}$-assisted photodegradation of pharmaceuticals - a review. Central European Journal of Chemistry, 10(4): 989-1027.

Treml, J.; Smejkal, K. 2016. Flavonoids as Potent Scavengers of Hydroxyl Radicals. Comprehensive Reviews in Food Science and Food Safety, 15(4): 720-738.

Trovó, A.G.; Nogueira, R.F.P.; Agüera, A.; Alba, A.R.F.; Malato, S. 2012. Paracetamol degradation intermediates and toxicity during photo-Fenton treatment using different iron species. Water Resource, 46(16), 53745380 . 
Valdez, H.C.A.; Jiménez, G.G.; Granados, S.G.; Léon, C.P. 2012. Degradation of paracetamol by advance oxidation processes using modified reticulated vitreous carbon electrodes with $\mathrm{TiO}_{2}$ and $\mathrm{CuO} / \mathrm{TiO}_{2} / \mathrm{Al}_{2} \mathrm{O}_{3}$. Chemosphere, 89(10), 1195-1201.

Xiong, P.; Hu, J. 2012. Degradation of acetaminophen by UVA/LED/TiO2 process. Separation and Purification Technology, 91(1): 89-95.

Wang, A.; Zhang, Y.; Zhong, H.; Chen, Y.; Tian, X.; Li, D.; Li, J. 2018. Efficient mineralization of antibiotic ciprofloxacin in acid aqueous medium by a novel photoelectro-Fenton process using a microwave discharge electrodeless lamp irradiation. Journal of Hazardous Materials, 342(1): 364-374.

Yavas, A.Z.; Mizukoshi, Y.; Maeda, Y.; Ince, N.H. 2015. Supporting of pristine $\mathrm{TiO}_{2}$ with noble metals to enhance the oxidation and mineralization of paracetamol by sonolysis and sonophotolysis. Applied Catalysis B: Environmental, 172-173(Ago.): 7-17.

Yavas, A.Z.; Ince, N.H. 2016. Enhanced photo-degradation of paracetamol on n-platinum-loaded $\mathrm{TiO} 2$ : The effect of ultrasound and ${ }^{\circ} \mathrm{OH} / \mathrm{hole}$ scavengers. Chemosphere, 162(Nov.): 324-332. 